\section{Modelo teórico para el alineamiento estratégico en las organizaciones, un valor oculto en la educación}

\author{
Theoretical model for strategic alignment in organizations, a \\ hidden value in education
}

\begin{abstract}
RESUMEN
La problemática del alineamiento estratégico, constituye un valor agregado al contenido que se presenta, ya que se puede apreciar, cómo el enfoque estratégico que va más allá de la planificación y penetra a todas las funciones directivas, exigiendo una mirada integradora a todo el proceso de dirección. Resulta de sumo interés, cómo se integra también la problemática del alineamiento a la utilización de los modelos de excelencia y de mejora continua, de manera que la calidad educativa sea un elemento sustancial de las estrategias.

El significativo interés que existe por la capacidad de alineamiento, como un elemento de la cultura organizacional y las capacidades dinámicas de absorción, como elemento desencadenante de las capacidades dinámicas de coordinación, tan necesarias para lograr una cultura de alineamiento en las organizaciones. Por ello, imprime un valor especial la contextualización del tema porque se adapta el resultado teórico a las condiciones de las organizaciones económicas y educativas, ofreciendo respuesta a la interrogante básica de esta obra, es decir: ¿Cuál es el valor oculto de las organizaciones mexicanas? lo que constituye un valor adicional que ha proporcionado el alineamiento entre los esfuerzos de esta labor cooperada. Por todo ello, el artículo va a llenar un espacio importante en la literatura científica y académica, dedicada al estudio del enfoque estratégico en la gestión de las organizaciones de México. Así, el proceso de coordinación en el cual se identifican los factores de alineamiento y se crean las condiciones organizativas necesarias para que se haga efectiva la cooperación e integración entre los recursos, las capacidades, los procesos y los implicados en la implementación de la estrategia que demande la manifestación de estos factores.
\end{abstract}

Palabras claves: Modelo teórico; alineamiento estratégico; organización y educación.

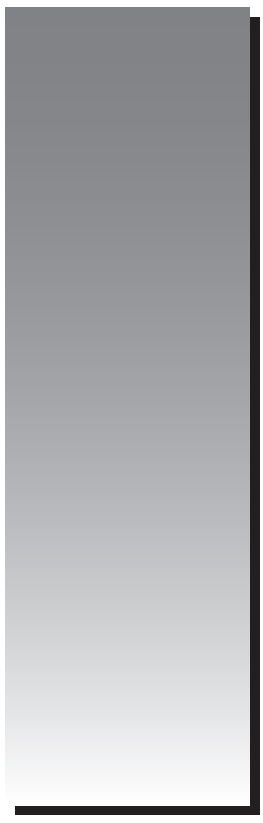

Lorenzo Salgado García ${ }^{1}$

l_salgadomx@yahoo.com.mx

Juan Victoriano Castillo Maza $^{2}$

Juan5705@yahoo.es

Fernando Javier García Colina $^{3}$

colinag2001@yahoo.es

${ }^{1}$ Benemérita de la Universidad Autónoma de Puebla, Facultad de Economía. México

${ }^{2}$ Universidad Nacional Mayor de San Marcos, Facultad de Ciencias

Administrativas. Lima, Perú

${ }^{3}$ Universidad Autónoma de Tlaxcala, Facultad de Ciencias Económico Administrativas. México 


\begin{abstract}
The issue of strategic alignment is an added value to the content presented, as you can see, how the strategic approach goes beyond planning and penetrates all management functions, demanding an integrated look at the whole process of address. It is of great interest how the issue of alignment to the use of models of excellence and continuous improvement, so that the quality of education is a substantial element of the strategy is also integrated.

The significant interest that exists for capacity alignment, as an element of organizational culture and dynamic absorption capacities, as an element triggering the dynamic capabilities of coordination, as necessary to achieve a culture of alignment in organizations. Therefore, it prints a special value contextualize the topic because the theoretical result is adapted to the conditions of economic and educational organizations, providing answer to the basic question of this work, namely: What is the hidden value of the Mexican organizations ? Which is an additional value that has provided the alignment between the efforts of the cooperated work.

Therefore, the article will fill an important gap in the scientific and academic literature devoted to the study of the strategic approach in the management of organizations of Mexico. Thus, the coordination process in which alignment factors are identified and organizational conditions are created so that becomes effective cooperation and integration between the resources, capabilities, and processes involved in the implementation of the strategy demands the manifestation of these factors.
\end{abstract}

Keywords: Theoretical model; strategic alignment; organization and education.

\section{INTRODUCCIÓN}

Las investigaciones recientes demuestran, lo importante que resulta para el desempeño de las organizaciones como funcionar, con arreglo al efecto obtenido de coordinar esfuerzos, recursos y capacidades de actividades diferentes hacia un fin común. Los ejecutivos de la oficina central, deben determinar cómo añadir valor a su conjunto de unidades de negocio, para que el total sea superior a la suma de sus partes por separado.

Las organizaciones complejas están compuestas de muchas actividades, cada una de las cuales ofrece un resultado, alcanzado mediante la gestión de sus competencias específicas, recursos y el apoyo, directo e indirecto, de otras actividades del grupo. (Mintzberg y Lampel, 1999; Porter y Siggelkow, 2008). Dicho esfuerzo, se integra a los resultados globales del grupo y este se constituye en posicionamiento en el mercado, preferencia de clientes y valor para los accionistas. Sin embargo, la experiencia de más de 15 años de investigaciones y consultorías, nos han permitido identificar llamativas reservas de eficiencia en la gestión empresarial.
El trabajo dedica en un primer momento, al análisis de las principales manifestaciones de dicho comportamiento a partir de un profundo estudio realizado en varias empresas y otras organizaciones mexicanas en los últimos cinco años. Una de las principales incongruencias encontradas en el despliegue estratégico, del $88 \%$ de las empresas estudiadas radica en la débil descripción de dicha estrategia y, en grado similar, la falta de una proyección que integre y optimice los esfuerzos hacia una prioridad establecida.

En todos los casos, la implementación hasta la base del modelo estratégico definido se desarrolló a partir de las orientaciones, objetivos, políticas y líneas de trabajo definidas por una idea central. Sin embargo, los resultados alcanzados no estuvieron a la altura de las capacidades poseídas para ello. Todo lo anterior, indica que, la necesidad del conocimiento de los factores que favorecen o inhiben la producción de sinergias provenientes del alineamiento en la implementación de las estrategias en las organizaciones, para enfocar los sistemas de control estratégico sobre estos aspectos. 
El campo de estudios se enmarca en el proceso de dirección estratégica de las empresas y se particulariza en los factores que condicionan el alineamiento estratégico y su correlación con los resultados de las mismas. Este trabajo se incluye entre los que, asumen el alineamiento estratégico como un complejo proceso holístico de coordinación para alcanzar el éxito y se encarga de ofrecer criterios científicamente comprobados de que los factores que inhiben o favorecen el alineamiento estratégico en las organizaciones permiten influir en los resultados alcanzados por las mismas, como es el caso de la Educación.

Esta hipótesis general implícita, está fundamentada por los resultados de investigaciones que demuestran que los mecanismos de coordinación y fundamentalmente los de alineamiento estratégico, permiten influir sobre los resultados alcanzados, en forma de un valor adicional al producido por el funcionamiento de las Unidades Estratégicas de Negocio, identificado como los resultados de la sinergia que se logra por dichos procesos de coordinación (Mintzberg y Waters, 1985; Menguzzato y Renau, 1995; Porter, 1996a; Mintzberg y Lampel, 1999; Kaplan, Norton y Atkinson, 2001; Kramer y Porter, 2006; Raynor, 2007; Porter, 2008).

De esta manera, la eficacia con que se manejen los factores que inhiben o favorecen el alineamiento estratégico en las empresas mexicanas, permite influir sobre los resultados globales de las mismas e identificar el efecto donde se manifestarán las diferentes formas de sinergia. Se ofrece, un análisis de la cultura organizacional y su relación con los niveles de alineamiento alcanzados por las empresas estudiadas, lo que apunta a que sí, la organización no está comprometida con una cultura de cooperación, tendrá dificultades para alcanzar un valor derivado de la organización. La investigación empírica realizada se ha dirigido a la alta dirección de empresas y organizaciones mexicanas de diferentes actividades y sectores: transporte, alimentación, almacenamiento, publicidad, salud, educación, consultoría, entre otras.

Se presenta un modelo teórico general que permite ofrecer una descripción detallada del sistema de relaciones que se producen entre las diferentes variables y factores que entran en juego en los procesos de Alineamiento Estratégico como principal fuente de sinergias debida a los procesos de coordinación, contextualizándolo al caso de las organizaciones. De esta manera, el lector tiene en sus manos, un modelo de gestión y evaluación del Alineamiento Estratégico como principal propuesta para la educación, elementos que permiten absorber resultados derivados del proceso de coordinación e incrementar directa y definitivamente los niveles de competitividad. Así, se describe una metodología para el despliegue y los procedimientos inherentes a ella, con la definición de las variables a considerar, así como la definición del sistema de hipótesis que son contrastadas con posterioridad a partir del estudio realizado.

A partir de este resultado, se justifica la metodología que se emplea, así como los procedimientos utilizados para ofrecerle validez científica a las diferentes inferencias que se están realizando, tomando en consideración la diversidad que se encuentra en el conjunto de empresas estudiadas, cas diferente entre ellas. Se define así, como universo de la investigación un sistema de empresas, caracterizadas por un conjunto de indicadores que permitió, a través de la aplicación del método multivariante de los clústeres, establecer los grupos que presentaban características semejantes en cuanto a un conjunto de variables. Esto permitió aislar la influencia de los factores de alineamiento estratégico, del resto de los factores tenidos en cuenta para la obtención de resultados por dichas empresas.

El resultado fundamental del trabajo, es el establecimiento de un modelo que permite definir los indicadores para evaluar el Alineamiento Estratégico a partir de la integración, en un sistema evaluativo, del conjunto de instrumentos utilizados. Esto, deja sentadas las bases para desarrollar procesos de innovación y adaptación que permitan la utilización de estos resultados para la definición de un sistema de mejora continua que, al ser aplicado a la gestión de estos procesos de coordinación, permite alinear a la organización con la estrategia de una forma continuada y en un proceso de mejora continua como los que son caracterizados por la generación de procesos sinérgicos. 
El trabajo invita a la reflexión sobre un conjunto de ideas, con nuestro razonamiento y arribar a conclusiones propias, destinadas a hacerle protagonista, a partir de su propia experiencia, de una transformación de su negocio, su mentalidad administrativa y de su sistema de trabajo en un equipo totalmente evolucionado a un nivel superior de competitividad. Sean bienvenidos también académicos, estudiantes, investigadores de la educación y emprendedores a este recorrido por una nueva visión de creación de valor: el alineamiento estratégico. Todo el proceso se propone analizar un modelo teórico general, que permite establecer las pautas para la identificación de los factores que produce el alineamiento estratégico y cómo éstos son gestionados por el equipo directivo. A partir del mismo, se describe la forma de manifestarse el alineamiento, según sea cada factor en las organizaciones educativas.

\section{Factores genéricos de alineamiento estratégico.}

Existen diferentes estudios, donde se expresan las tendencias y concepciones asumidas en la literatura, se pueden identificar y resumir, un conjunto de factores genéricos que provocan alineamiento, asumiendo estos, como aspectos determinantes dentro del proceso de coordi- nación. La identificación y determinación de estos factores se realizó mediante los siguientes pasos: Contrastación de criterios autorales sobre los elementos condicionantes de la estrategia y su relación con la estructura. Las principales posiciones sobre este tema esclarecen la subordinación de los elementos estructurales a los requerimientos estratégicos. Los análisis de los criterios autorales relacionados con el alineamiento estratégico, se pueden apreciar en la figura 1.

Estos factores de alineamiento en el proceso de coordinación se manifiestan en la estructura organizativa de la organización, pero en aquellas subdivisiones que tienen un determinado significado para la Estrategia (Unidad Estratégica de Negocios; USC y UAC). Estos procesos de coordinación pueden aplicarse dentro de las Unidades Estratégicas, en las de Soporte o de Apoyo o pueden aplicarse como procesos de coordinación entre distintas Unidades Estratégicas de Negocio. En todo caso estos procesos de coordinación deben ser promovidos por la corporación ya bien sean procesos de coordinación interna o entre Unidades Estratégicas. Este proceso termina produciendo un estado de Alineamiento en la organización. Existen autores que llegan a plantear que cuando éste estado, a través de un proceso de aprendizaje e interiorización se convierte en

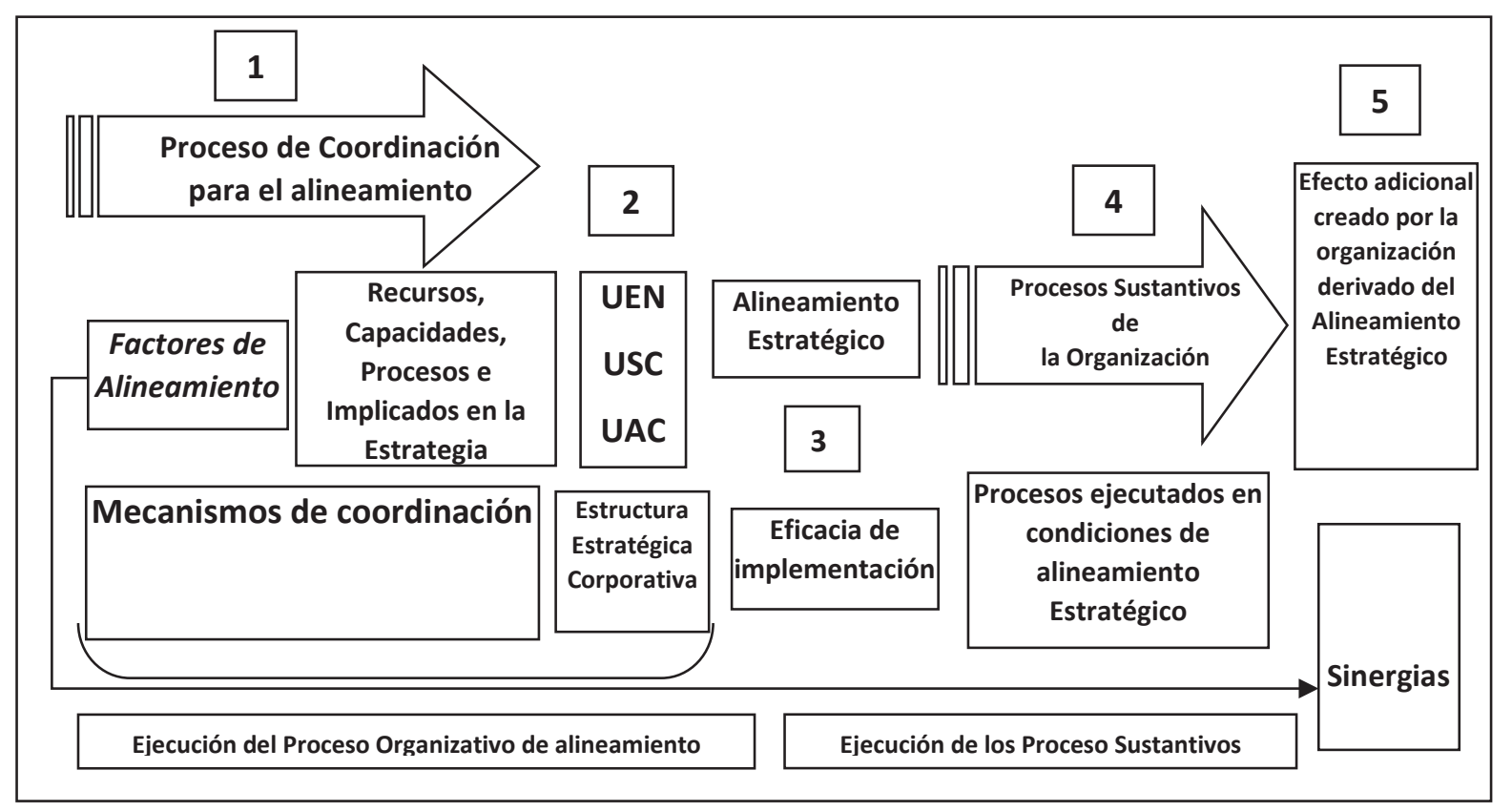

Figura 1. Modelo general de alineamiento estratégico en las organizaciones Adaptado de Alineamiento Estratégico por Oliek (2015). 
una convicción de los miembros de la organización, puede ser reconocido como un valor compartido (González, 2013).

En este estado la organización acomete la ejecución de sus procesos sustantivos en función de su misión, sobre la base de un estado superior de coordinación. Esto significa que dichos procesos sustantivos son llevados a cabo en condiciones de alineamiento estratégico. Esta fase de coordinación se enmarca dentro del proceso estratégico, y está referida solo a las relaciones y acciones que se establecen en función de la estrategia de las unidades de negocio y de la estrategia general planteada.

Las principales posiciones y criterios sobre la capacidad de generar resultados superiores a partir de mejoras entre relaciones organizativas, que impliquen ahorro de recursos y tiempo, aprovechamiento de capacidades o generación de otras nuevas, incremento en el rendimiento, incremento en la eficiencia; asumiendo la estrategia, como factor común; simplificación y jerarquización de las posiciones y criterios según la lógica del alineamiento estratégico como proceso. Esto, supone un enfoque sistémico por cuanto el alineamiento estratégico como proceso transita por los diferentes niveles de la organización a través de los puntos que lo movilizan, en adelante: factores. Es por ello, que, son identificados como acciones de coordinación entre los recursos, las capacidades, los procesos y los implicados en la estrategia. El ajuste o complemento de los factores genéricos según el modelo general. El resultado del análisis anterior se relaciona en el siguiente grupo de diez factores genéricos, que intervienen en el proceso de alineamiento estratégico.

Alineamiento entre Estrategia y Estructura. (Menguzzato y Renau, 1995; Mintzberg y Lampel, 1999; Venkatraman y Gering, 2000; Mintzberg y Rose, 2003; Prahalad y Hamel, 2005; Kaplan y Norton, 2006; Scherpereel, 2006; Ezingeard, et al., 2007; Shpilberg, et al., 2007; Porter, 2008). Es ampliamente reconocido en la literatura, desde los clásicos hasta los más actuales trabajos, el papel decisivo de la estructura en la consecución de los objetivos de la organización, pues a través de ella se materializan. Los autores citados son solo ejemplos del tratamiento de este tema, desde varios en- foques y posiciones. La coordinación entre la estrategia y la estructura, es la encargada de desagregar las funciones y responsabilidades requeridas por la estrategia. Este factor establece y mantiene actualizados los vínculos entre la estructura y la estrategia.

El tema abordado con énfasis aquí, es el papel de los canales de comunicación, como lubricante entre la estructura y la estrategia. La información, debe constituir un reflejo del curso de la estrategia a través de la organización. La coordinación, por tanto, debe atender también los pormenores de cada unidad estratégica y de apoyo o soporte en el cumplimiento de su parte dentro de la estrategia.

El alineamiento de intereses (Stakeholders): Coordinación de intereses entre la organización y el financiamiento externo, accionistas, proveedores, determinados clientes, aliados, etc. (Mintzberg y Rose, 2003; Kaplan y Norton, 2006; Carneiro, 2007; Henriques, 2007; Hua, 2007; Scholey, 2007; Shpilberg, et al., 2007; Porter y Siggelkow, 2008). Este factor es el resultado de la coordinación entre la organización y sus implicados. Este es un factor que se trata desde la importancia de mantener una posición atractiva y confiable ante el financiamiento externo. También se describen relaciones materializadas en apoyo y respaldo a determinados programas de desarrollo, de impacto social o medioambiental por parte de los accionistas u otros agentes externos. Otras posiciones destacan la importancia de representar y fortalecer los intereses de instituciones administrativas como gobierno, ministerios, fundaciones, entre otras. En cualquier caso, la misión de la organización debe respaldar los intereses de los implicados y servir de base para objetivos comunes. Por tanto, la dirección debe velar por el estado de los intereses entre las partes y gestionar mecanismos que permitan la coherencia entre ambos y su manifestación a través de la estrategia. Este factor relaciona la estrategia con el entorno, lo que implica que el alineamiento debe ir más allá de los límites de la organización. Gestionar los procesos alineados con los intereses de inversionistas contribuye a atraer capital, tecnología y mercado hacia la corporación.

Como se vio en la primera parte, la organización debe además prestar implicados en la 
estrategia. Unidades de negocio diferentes, e incluso las actividades de las unidades de apoyo y soporte pueden compartir clientes y proveedores. La coordinación entre ellos por tanto permite aprovechar y compartir capacidades instaladas, reducir costos y tiempo, aportando a la eficiencia de los procesos. Se fomenta además la atención particularizada a los clientes y las garantías entre los proveedores lo que aporta ventajas antes la competencia y contribuye en términos de eficacia.

El alineamiento de identidades: (Kaplan y Norton, 2006; Kearns y Sabherwal, 2006; Scherpereel, 2006; Sledgianowski, Luftman y Reilly, 2006). Este factor es tratado en la literatura desde las oportunidades que genera una organización si coordina y comparte marcas, franquicias, lemas, entre otros rasgos de identidad. De una parte, permite aprovechar espacios ganados por otras unidades de negocio, para abordar nuevos mercados o acceder a nuevas tecnologías. De esta manera se reducen gastos de publicidad y promoción, se aprovechan capacidades instaladas y se promueven servicios integrales. Por otra parte, permite inculcar y promover en los clientes y proveedores una imagen de cohesión y trabajo en equipo, lo que fortalece la posición competitiva y eleva la confianza de los proveedores, incrementa cuotas de mercado, afianza la fidelidad del cliente y se acceden a ofertas especiales y de calidad.

La coordinación interna. Coordinación e integración hacia dentro de las Unidades Estratégicas. (Venkatraman y Gering, 2000; Kaplan y Norton, 2006; McLean, 2006; Sledgianowski, et al., 2006; Paladino y Williams, 2008). Este factor se refiere a la coordinación de los procesos desarrollados en cada estrategia particular. Es una coordinación hacia dentro de las Unidades donde se desarrollan dichas estrategias. La literatura fundamenta este criterio desde la especialización de las actividades internas de la unidad estratégica, diseñada para cumplir un objetivo específico dentro de la misión de la organización. Este factor hace hincapié en el fortalecimiento y sistematización de los elementos que componen las actividades de cada responsable, lo que conlleva al fortalecimiento de cada estrategia.

Las unidades estratégicas emplean recursos, capacidades y tiempo en el desarrollo de sus procesos. Trabajos como los anteriores y otros como los de Henderson y Venkatraman (1993), Mintzberg y Waters $(1982,1985)$ o Porter (2008), describen, a partir de modelos teóricos o validaciones empíricas, la obtención de ventajas competitivas en la medida en que los insumos son óptimamente distribuidos y aprovechados en la cadena de valor de la unidad estratégica. Este, por ejemplo, es uno de los pilares de una estrategia de liderazgo en $\operatorname{costos}^{1 .}$ Así, se reconoce también, la contribución de esta coordinación a la eficacia de la unidad estratégica y de la organización pues se potencia la propuesta de valor al cliente desde la consolidación de los atributos del producto o servicio y la incorporación otros más exigentes.

El alineamiento interno. (Venkatraman y Gering, 2000; Fuller y Vassie, 2002; Kramer y Porter, 2006; Ezingeard, et al., 2007; Conz, 2008; Dong, Liu y Yin, 2008; Huang, et al., 2008). La coordinación entre unidades estratégicas presupone la coordinación entre estrategias diferentes dentro de la organización. Constituye el ejemplo más abordado en la literatura referida a la dirección estratégica en general, y en particular al alineamiento estratégico. Este factor es tratado fundamentalmente desde la diversificación de las actividades de una organización.

En los primeros párrafos, se analizó la importancia de la coordinación de los distintos procesos desarrollados en la organización con el propósito no solo de optimizar el uso de recursos, sino de obtener un resultado superior a partir de la efectiva integración de las diferentes estrategias particulares. La coordinación entre unidades estratégicas, contribuye a complementar procesos y compartir recursos, capacidades e implicados a partir de las diferentes cadenas de valor.

Alineamiento entre Unidades Estratégicas y de Apoyo o Soporte. (Shakun y Melvin, 1978; Porter, 1996a; Lynch, 1998; Mintzberg y Lampel, 1999; Madhok, 2002; Korver y van Ruler, 2003; Rees y Porter, 2004; Kaplan y Norton, 2006; Griffy-Brown y Chun, 2007). Otro factor identificado en la revisión bibliográfica es la coordinación que debe existir entre los proce-

1 Este tema se aborda ampliamente en trabajos como los de Porter (1996, 2006) entre otros. La estrategia de liderazgo en costos requiere de fórmulas organizativas que contribuyan a fomentarla como una ventaja competitiva. 
sos clave o actividades primarias o sustantivas y las actividades o servicios complementarios a estas, derivados de las unidades de apoyo de la organización. Las actividades o servicios secundarios, consumen recursos en función de complementar las actividades que realizan las unidades estratégicas. La literatura concede gran importancia a la forma en que se coordinan estas actividades para aprovechar economías generadas por los costos fijos, capacidades instaladas, actividades especializadas, asesoría entre otras, lo que se constituye en fuente de eficiencia.

Cada vez con mayor frecuencia, las actividades secundarias son externalizadas (Mintzberg y Lampel, 1999; Raynor, 2007; Crump, 2008; Agarwal y Selen, 2009; Diana y Samson, 2011). Con el propósito de eliminar costos asociados a ellas como depreciación o mano de obra y aprovechar la experiencia de agentes externos especializados. En cualquier caso, la coordinación entre estas actividades y las actividades primarias constituye un aspecto de vital importancia dentro del alineamiento, pues contribuye a la optimización de recursos y tiempo a través de un servicio compartido. Un servicio integral como el transporte o la selección y capacitación del personal, coordinado desde la alta dirección para los diferentes procesos, facilita la atención y tratamiento a las prioridades de la estrategia y el equilibrio entre unidades estratégicas.

Alineamiento e integración entre procesos. (Vicens, 2004; Mehta y Hirschheim, 2007; Rahman y Eisner, 2007). La coordinación entre procesos es abordada en la literatura haciendo énfasis en las relaciones entre unidades estratégicas, pero en ocasiones los procesos trascienden esas fronteras. Varias estrategias pueden compartir uno o varios procesos diferentes, de los cuales se nutre a lo largo de las cadenas de valor relacionadas. (Porter, 1996a). Es por ello, que, la coordinación entre los procesos es también determinante dentro del alineamiento estratégico. Este tratamiento es abordado tempranamente desde la dirección por procesos y argumentado por varios autores (Mintzberg y Lampel, 1999; Sasiain, 2004; Snider, 2006; Tallon, 2007). Los sistemas de calidad, por ejemplo, se circunscriben generalmente a los procesos de la organización, y monitorean los estándares de comportamiento de cada parte de ellos, sin embargo, es reconocido que la calidad en las organizaciones no es la suma de la calidad de sus procesos, sino de su integración como sistema. La coordinación entre procesos contribuye a fomentar soluciones integrales al aprovechar las capacidades específicas de cada uno, dentro y fuera de la unidad estratégica.

Alineamiento entre la estrategia y la actividad administrativa. (Mintzberg y Lampel, 1999; Kaplan y Norton, 2004; Raymond y Croteau, 2006; Sledgianowski, et al., 2006; Ezingeard, et al., 2007). En la medida que la dirección conozca las prioridades de la estrategia y el potencial de la organización que dirige podrá conducir con mayor eficacia el proceso de gestión hacia todas las partes esta. Las actividades administrativas deben tener un impacto directo en los procesos sustantivos de sus unidades estratégicas.

Tanto en la definición de alineamiento estratégico asumida, como en el modelo teórico planteado, la dirección es reconocida como la máxima responsable del alineamiento pues es responsable de facilitar la consecución e integración de los procesos y hacerlos notar en su entorno socio-económico a través de la interacción entre accionistas, representantes de otros organismos, clientes, proveedores, medios de comunicación y las unidades estratégicas que dirige. Este factor, es tratado por tanto, desde la forma en que el trabajo de la dirección constituye el motor impulsor de la estrategia y orienta los esfuerzos de su organización sobre las prioridades de la misma.

En cuanto al alineamiento de activos intangibles. Lograr un aprovechamiento coordinado de los Activo Intangibles. (Kaplan y Norton, 2006; Greiner, Böhmann y Krcmar, 2007; Wegmann, 2007; McDonough, Zack, Lin y Berdrow, 2008). Este factor se identifica a partir de la implicación de los activos intangibles en el logro de la eficacia. Aquí se refuerza la idea de las capacidades dinámicas.

El alineamiento de los recursos humanos, manifestado en intereses individuales y colectivos, el aprovechamiento de las tecnologías y la innovación, el conocimiento compartido, etc., son ejemplos citados y descritos sobre este factor en la literatura. La coordinación e integra- 
ción de la información y el conocimiento con la estrategia se traducen en mejora de los procesos de todas las unidades a partir de compartir e integrar las mejores prácticas, reducir costos de la duplicación de información, reducir tiempo en actividades repetidas, reducir trámites con clientes y proveedores, etc. Por lo tanto, el trabajo de las personas, tanto individual como colectivo, los resultados se manifiestan en la medida que se vinculen y comprometan con el resultado global de la organización y no con procesos particulares o unidades específicas.

Alineamiento de las actividades de control (Lawson, Stratton y Hatch, 2003; Kearns y Sabherwal, 2006; McDonough, et al., 2008). La literatura consultada describe y argumenta actividades de control, dirigidas a monitorear y ajustar consecuentemente, el funcionamiento efectivo de la estructura en relación con la estrategia, reconociendo la necesidad de coordinar las actividades de control de los diferentes procesos, para garantizar su eficacia. Una misma actividad de control puede brindar información sobre varios procesos clave, si estos son monitoreados como un sistema, en donde se comparten recursos, capacidades y tiempo.

La coordinación entre los sistemas y sus consecuentes actividades de control, contribuye a reducir información innecesaria, garantizar la oportunidad y pertinencia de la misma, acortar el tiempo entre la desviación y la acción correctiva y disminuye el costo derivado del control. Todos los factores enunciados tienen un carácter organizativo, enfocado hacia la actividad de coordinación que debe realizar la dirección para mantener a su organización alineada con su estrategia. Algunos factores pueden parecer incluidos en otros, pero han sido identificados por separado para hacer hincapié en las características específicas de cada elemento participante en el alineamiento y su manifestación según la forma en que se coordine.

La capacidad de estos factores para generar alineamiento, dependerá de la medida en que cada uno de los procesos que llevan asociados se ejecute de manera eficiente, dando lugar a múltiples medidas de carácter organizativo para que los mismos se materialicen. En cada organización estos factores genéricos tienen mecanismos de coordinación y formas parti- culares que los hacen manifestarse, en dependencia de las características de los procesos sustantivos que se desarrollen. Es por ello, que estos factores deben ser contextualizados a las características de los procesos específicos que en ellas se desarrollen.

\section{Contextualización de los factores de ali- neamiento a la realidad mexicana.}

La contextualización de los factores genéricos, entendida como la forma de manifestarse cada factor en la empresa independientemente de su tamaño o nivel de actividad y su pertinencia dentro del contexto empresarial mexicano, se desarrolló en los dos pasos siguientes: La opinión de los autores, apoyada en entrevistas a directivos y empresarios y la actividad consultora y académica. Este paso dio lugar a una relación de valoraciones, que representan la manifestación de los factores de alineamiento estratégico, en una versión inicial.

En consecuencia, la opinión de un grupo de expertos, encargado de confirmar el grado de contextualización y representatividad de los factores de alineamiento estratégico, según las valoraciones derivadas del paso anterior. Se seleccionaron expertos de varias universidades, unidades consultoras, contadores públicos, asesores jurídicos y personal de gobierno, hasta completar un total de 30, según los requerimientos de Birdir y Pearson (2000) para este tipo de estudios, que reunieran las características siguientes:

Más de 20 años de experiencia en la práctica empresarial mexicana. Responsabilidades en la coordinación de procesos en su desempeño profesional. La contextualización de los factores de alineamiento estratégico se realizó aplicando el método de expertos en dos rondas. Por ello, en la primera ronda los expertos opinaron sobre la pertinencia de cada factor contextualizado y ofrecieron criterios que permitieron depurar los 27 definidos. Para validar las opiniones recogidas se asumieron las hipótesis siguientes:

$\mathrm{H}_{0}$ : No existe acuerdo entre los expertos encuestados.

$\mathrm{H}_{1}$ : Existe acuerdo entre los expertos encuestados.

El nivel de acuerdo en las opiniones se representa en la siguiente tabla: 
Tabla 1

Estadísticos de contraste. Prueba $W$ de Kendall, primera ronda. Contextualización.

\begin{tabular}{lc}
\hline $\mathrm{N}$ & 30 \\
\hline $\mathrm{W}$ de Kendall &, 722 \\
\hline Chi-cuadrado & 563,155 \\
\hline gl & 26 \\
\hline Sig. asintót. &, 000 \\
\hline
\end{tabular}

Fuente: elaboración propia, con los datos antes señalados.

El coeficiente de concordancia de Kendall $W=0,722>0$ es significativo. El valor de significación es menor que el nivel de significación $\alpha=0,05$ por lo que se rechaza la hipótesis de nulidad, es decir, existe acuerdo entre los expertos.

Se valoraron las opiniones a través de la mediana y la moda, buscando que la mayoría de las opiniones coincidiera con el valor central, por cada factor contextualizado. Se consideraron válidos los que consiguieron una evaluación superior a 4 en la escala de 1 a 5 utilizada.

Las principales consideraciones de los expertos para los factores con menor evaluación se resumen en:

Repetido en otro factor.

Difícil de medir.

Limitado en su alcance, por no incluir determinado criterio.

\section{Escasa explicación.}

Con las consideraciones emitidas por los expertos, se reformularon algunos factores y se realizó una segunda ronda, incorporando los criterios recibidos.

Asumidas las mismas hipótesis para la segunda ronda se comprobó el nivel de acuerdo entre los expertos:

Tabla 2

Estadísticos de contraste. Prueba $W$ de Kendall, segunda ronda. Contextualización.

\begin{tabular}{lc}
\hline $\mathrm{N}$ & 30 \\
\hline $\mathrm{W}$ de Kendall &, 108 \\
\hline Chi-cuadrado & 77,974 \\
\hline gl & 21 \\
\hline Sig. asintótica &, 000 \\
\hline
\end{tabular}

Fuente: elaboración propia, con los datos antes señalados.

Nuevamente el valor de significación es menor que el nivel de significación $\alpha=0,05$ por lo que se rechaza la hipótesis de nulidad, es decir, sigue existiendo acuerdo entre los expertos.
Se consideraron válidos los factores cuyas moda y mediana consiguieron una evaluación superior a 4 en la escala de 1 a 5 utilizada. Esta vez, las evaluaciones de los expertos, para todos los factores estuvieron entre 4 y 5 , lo que mostró una mayor pertinencia de los criterios propuestos. Como resultado, quedaron establecidos, los siguientes 21 factores contextualizados a las empresas mexicanas (Ver tabla 3).

A través de estos pasos se logró determinar y corroborar, cuáles son las manifestaciones concretas del alineamiento como proceso en las empresas mexicanas. La evaluación de esta manifestación, brinda información acerca de cómo se gestiona el alineamiento a partir del modelo propuesto. El modelo teórico diseñado, representa una relación entre los factores genéricos de alineamiento y los resultados obtenidos por la organización, según el caso. La contextualización de estos factores en las condiciones, permite poner a prueba esta relación a través de la evaluación de los mismos en varias organizaciones y su posterior correspondencia con los resultados de cada una.

Las posiciones asumidas sobre las capacidades dinámicas y la cultura y su relación con el alineamiento dependen de la relación anterior. Ha quedado demostrado teóricamente que el alineamiento se convierte en una capacidad dinámica de coordinación una vez que el proceso de alineamiento sea consciente y planificado a través de los factores de alineamiento establecidos. Esto implica que la confirmación definitiva dependerá de la veracidad de la relación entre estos y los resultados. Una vez confirmada esta relación, entonces se confirmará que el alineamiento estratégico, al convertirse en una capacidad dinámica, la organización incorpora esa práctica a su conjunto de valores compartidos, y por tanto a su cultura. De esta manera, el alineamiento estratégico se convierte en un valor de la organización para la educación.

Luego, es necesario demostrar la relación entre los factores de alineamiento contextualizados, como manifestación de los factores genéricos en las empresas mexicanas y los resultados alcanzados por éstas. Este modelo funciona para las organizaciones económicas, como para las organizaciones educativas. 
Tabla 3

Factores contextualizados

\begin{tabular}{|c|c|}
\hline $\begin{array}{l}\text { Factor de alineamiento } \\
\text { estratégico }\end{array}$ & Manifestación de cada factor de alineamiento \\
\hline \multirow{2}{*}{$\begin{array}{l}\text { I. Coordinación de la es- } \\
\text { trategia y la estructura. }\end{array}$} & $\begin{array}{l}\text { Descripción de la estrategia de manera que cada elemento de la estructura conozca el lugar que ocupa en } \\
\text { su cumplimiento. }\end{array}$ \\
\hline & $\begin{array}{l}\text { Coordinación entre la estrategia, las áreas de resultados clave y los objetivos y los elementos estructurales } \\
\text { de la empresa. }\end{array}$ \\
\hline \multirow{4}{*}{$\begin{array}{l}\text { II. Coordinación de inter- } \\
\text { eses. }\end{array}$} & Coordinación de la estrategia con la de la alta dirección y/o el territorio. \\
\hline & Coordinación para la utilización de proveedores para varias Unidades Estratégicas. \\
\hline & Coordinaciones para la atención especializada a clientes. \\
\hline & $\begin{array}{l}\text { Coordinaciones para la creación y aprovechamiento de aliados externos en base a las prioridades de la } \\
\text { estrategia. }\end{array}$ \\
\hline $\begin{array}{l}\text { III. Coordinación de iden- } \\
\text { tidades. }\end{array}$ & $\begin{array}{l}\text { Existencia y funcionamiento de mecanismos de gestión de la empresa que permitan aprovechar el presti- } \\
\text { gio de alguna unidad estratégica en función de las demás. }\end{array}$ \\
\hline IV. Coordinación Interna. & $\begin{array}{l}\text { Coordinación para ofrecer servicios más integrales, complementados con los resultados de varias uni- } \\
\text { dades estratégicas. }\end{array}$ \\
\hline $\begin{array}{l}\text { V. Coordinación entre } \\
\text { unidades estratégicas. }\end{array}$ & $\begin{array}{l}\text { Coordinación para establecer sistemas de capacitación para asumir nuevas tecnologías, proceso producti- } \\
\text { vos, formas de hacer, etc. }\end{array}$ \\
\hline \multirow{2}{*}{$\begin{array}{l}\text { VI. Coordinación e entre } \\
\text { unidades estratégicas y de } \\
\text { apoyo o soporte. }\end{array}$} & $\begin{array}{l}\text { Coordinación de un servicio de las unidades de apoyo en unidades que presten servicio integral (ali- } \\
\text { mentación, transporte, seguridad, mantenimiento, etc.) a varias unidades estratégicas. }\end{array}$ \\
\hline & $\begin{array}{l}\text { Coordinación de los servicios de las unidades de soporte (Economía, RRHH, capacitación a directivos, } \\
\text { estimulación) }\end{array}$ \\
\hline $\begin{array}{l}\text { VII. Coordinación entre } \\
\text { procesos. }\end{array}$ & Formas organizativas de coordinación de los procesos principales. \\
\hline \multirow{2}{*}{$\begin{array}{l}\text { VIII. Coordinación entre } \\
\text { la estrategia y la actividad } \\
\text { administrativa. }\end{array}$} & $\begin{array}{l}\text { Grado en que los programas y contenidos de las actividades administrativas se corresponden con el con- } \\
\text { tenido y prioridades de las estrategias. }\end{array}$ \\
\hline & $\begin{array}{l}\text { Nivel de coordinación entre los objetivos, estrategias y sus indicadores a los distintos niveles hasta la base. } \\
\text { Forma en que se reflejan las estrategias, en los planes de resultados individuales, colectivos y de unidades } \\
\text { estratégicas y de apoyo. }\end{array}$ \\
\hline \multirow{5}{*}{$\begin{array}{l}\text { IX. Coordinación y aprove- } \\
\text { chamiento de los Activo } \\
\text { Intangibles. }\end{array}$} & Forma en que se organizan y coordinan las actividades de los factores. \\
\hline & Mecanismos de estimulación en función de los resultados del trabajo a mayor rendimiento mayor retribución. \\
\hline & $\begin{array}{l}\text { Formas de coordinación que permiten aprovechar el conocimiento de la organización para garantizar la } \\
\text { superación de todos los trabajadores }\end{array}$ \\
\hline & $\begin{array}{l}\text { Formas en que se propicia que se relacionen los directivos de mayor preparación en la superación del } \\
\text { personal. }\end{array}$ \\
\hline & $\begin{array}{l}\text { Formas de coordinación e integración de los distintos sistemas de dirección a través del establecimiento } \\
\text { de una plataforma común de Integración. }\end{array}$ \\
\hline \multirow{2}{*}{$\begin{array}{l}\text { X. Coordinación de la } \\
\text { supervisión y el control } \\
\text { entre áreas. }\end{array}$} & Formas de coordinación con los planes de control interno entre áreas. \\
\hline & l aporte individual y colectivo al resultado global de la empresa. \\
\hline
\end{tabular}

Fuente: elaboración propia, con los datos antes señalados.

\section{CONCLUSIONES}

Un modelo de alineamiento estratégico, permite teórica y metodológicamente arribar a las siguientes premisas: Primera. El modelo describe la relación entre los factores del proceso alineamiento y la obtención de resultados, por lo que, se puede derivar que, los factores de alineamiento son responsables de los resultados de la organización educativa. En el contenido del documento, se demostró cómo los resultados obtenidos por un sistema, no son consecuencia o efecto de un solo proceso o actividad, o de la simple manifestación de uno de sus componentes; sino de la interacción de todos ellos. De aquí se desprende que el alineamiento como proceso es solo uno de los generadores de resultados. Una organización con mayores recursos económicos y capacidades, unas más que otras; estaría en condiciones de obtener mayores resultados cuantitativos que la segunda. Sin embargo, los resultados cualitativos dependerán de la forma en que logre orientar sus esfuerzos hacia su estrategia. Por otra parte, toda organización, es la suma de factores financieros, materiales, tecnológicos y el trabajo de sus miembros, por lo que, el alineamiento como proceso es, solo uno de los elementos que influye en sus resultados. Las organizaciones, con idénticas proporciones de capital, materiales y tecnología educativa, situadas en entornos idénticos o muy similares, deberían obtener idénticas 
proporciones de resultado; sin embargo, no es así. La diferencia radica en la forma en que se coordinen las actividades de ellas. Para demostrar o cuantificar la relación entre los factores de alineamiento y los resultados de la organización, es necesario aislar el efecto del resto de los factores en dichos resultados.

Una segunda aproximación del modelo de alineamiento estratégico, son los factores genéricos hacen referencia a procesos de coordinación, formal o informal, que condicionan la obtención de resultados derivados del aprovechamiento en función de la estrategia, de los recursos, las capacidades, los procesos y los implicados en la estrategia de la organización. Estos procesos se gestionan a través de mecanismos de coordinación que le permitan a la dirección, alcanzar el estado deseado. El éxito en la gestión de cada factor depende de la eficacia con que se implementen dichos mecanismos. Por tanto, los factores genéricos, a partir del proceso de alineamiento estratégico, son capaces de influir notablemente en los resultados de una organización, cualesquiera que sean sus características.

Tercera: El alineamiento estratégico, como proceso gestionado a través de los factores genéricos, constituye un proceso pensado, mediante el cual, la dirección intencional la obtención de resultados superiores a partir de la disposición de sus recursos, capacidades, procesos e implicados en la estrategia. Como se evidencio a lo largo del texto, el alineamiento es gestionado en muchas organizaciones, por la capacidad de sus líderes para detectar un aprovechamiento potencial de recursos y tiempo en función de la estrategia. En tales casos, el alineamiento es ocasional e improvisado. Gestionar el alineamiento estratégico a partir de la utilización de los factores genéricos, se convierte en un proceso consciente y sistémico. Este proceso, mejorado constantemente a partir del aprendizaje ${ }^{2}$, se convierte en una capacidad dinámica de la organización, lógicamente difícil de superar (Zollo and Winter, 2002).

Por lo anterior expuesto, se corrobora que la identificación de estos factores genéricos de ali-

2 Las capacidades dinámicas surgen como resultado de un aprendizaje; constituyen métodos sistémicos de la empresa para modificar sus rutinas operativas. El grado en que los mecanismos de aprendizaje son sistemáticos, podrían ser considerados como capacidades dinámicas de segundo orden. neamiento, constituye una condición necesaria para que a través de un proceso de aprendizaje, la organización sea capaz de utilizarlos eficazmente en la obtención de un valor adicional, convirtiéndose así en una capacidad dinámica de la organización que resultará una ventaja competitiva difícil de imitar. La gestión reiterada y mejorada del alineamiento a través del aprendizaje, pasa a ser una conducta aprobada y asumida por los miembros de la organización, dada su capacidad demostrada para obtener resultados. Esta práctica reiterada, se convierte en conocimiento incorporado a la gestión de la organización. De esta manera puede llegar a constituir un valor compartido de la organización, incorporándose así a la cultura organizacional.

Los procesos en ocasiones se limitan al alcance de sus resultados, si no existe una acción coordinada. En tales casos, las organizaciones serían, en esencia, la suma de sus unidades estratégicas y de apoyo. Esta apreciación, tratada como una forma superior de coordinación, es reconocida como alineamiento estratégico desde varios puntos de vista, lo que constituye el núcleo conceptual de esta investigación.

\section{REFERENCIAS BIBLIOGRÁFICAS}

Carneiro, A. (2007). What is required for growth? Business Strategy Series.

Crump, B. (2008). How can we make improvement happen? Clinical Governance.

Diana, L. H. C. y Samson, C. S. (2011). Strategic repositioning in a dynamic environment. Library Management.

González-Solán, Ramón (2013) Universidad de Camagüey, cuba.

Hau-Siu, C. y Shan, S. L. (2007). Business Strategy, Organizational Culture, and Performance Outcomes in China's Technology Industry. HR. Human Resource Planning.

Hau-Siu, C. y Shan, S. L. (2007). Business Strategy, Organizational Culture, and Performance Outcomes in China's Technology Industry. HR. Human Resource Planning.

Henriques, C. (2007). Activity Alignment. Leadership Excellence.

Kaplan, R. S. (2008). Alcanzar su potencial. Harvard Business Review.

Kaplan, R. S. (Ed.). (2006). Alignment. Incrementando los resultados mediante el alineamiento estra- 
tégico de toda la organización (1ํㅡㄹ ed ed.). Barcelona: Gestión.

Kaplan, R. S. y Norton, D. P. (2000). Cómo utilizar el cuadro de mando integral: para implantar y gestionar su estrategia. Vol. 1.

Kaplan, R. S. y Norton, D. P. (2001). Building a strategy-focused organization. Ivey Business Journal.

Kaplan, R. S. y Norton, D. P. (2001b). The strategy-focused organization. Strategy \& Leadership.

Kaplan, R. S. y Norton, D. P. (2004). The strategy map: guide to aligning intangible assets. Strategy \& Leadership.

Kaplan, R. S. y Norton, D. P. (2005). El Balanced Scorecard: mediciones que impulsan el desempeño. Harvard Business Review.

Kaplan, R. S. y Norton, D. P. (2005). La organización focalizada en la estrategia: cómo implementar el Balanced Scorecard.Vol. 1. Gestión 2000.

Kaplan, R. S. y Norton, D. P. (2007). Usar el Balanced Scorecard como un sistema de gestión estratégica [artículo electrónico. Harvard Business Review.

Kaplan, R. S. y Norton, D. P. (2008). Dominar el sistema de gestión. Harvard Business Review.

Kaplan, R. S., Norton, D. P. y Atkinson, A. (2001). The strategy-focused organization: how balanced scorecard companies thrive in the new business environment. CMA Management..

Kaplan, R. y Norton, D. P. (2006). Alignment: cómo alinear la organización a la estrategia a través del Balanced Scorecard (Vol. 1): Gestión 2000.

Kearns, G. S. y Sabherwal, R. (2006). Strategic Alignment Between Business and Information Technology: A Knowledge-Based View of Behaviors, Outcome, and Consequences. Journal of Management Information Systems.

Kramer, M. R. y Porter, M. E. (2006). Estrategia y sociedad: el vínculo entre ventaja competitiva y responsabilidad social corporativa. Harvard Business Review.

Lawson, R., Stratton, W. y Hatch, T. (2003). The benefits of a scorecard system. CMA Management.

Lawson, R., Stratton, W. y Hatch, T. (2006). Strategic focus - does your scorecarding system have it? CMA Management.
Lawton, R. (2002). Align strategy \& measures with customer priorities. Quality Congress.ASQ's ...Annual Quality Congress Proceedings(Journal Article).

Lazonick, W. y Prencipe, A. (2005). Dynamic capabilities and sustained innovation: strategic control and financial commitment at Rolls-Royce plc. Industrial and Corporate Change.

Menguzzato, M. y Renau, J. J. (1995). La dirección estratégica de la empresa. Un enfoque innovador del management. (2da ed.): Prentice-Hall.

Mintzberg, H. (1987). The Strategy Concept Ii: another Look at Why Organizations. California management review.

Mintzberg, H. y Lampel, J. (1999). Reflecting on the strategy process. Sloan management review.

Mintzberg, H. y Rose, J. (2003). Strategic Management Upside Down: Tracking Strategies at McGill University from 1829 to 1980. Canadian Journal of Administrative Sciences.

Mintzberg, H. y Waters, J. A. (1982). Tracking Strategy in an Entrepreneurial Firm. Academy of $M a-$ nagement Journal.

Mintzberg, H. y Waters, J. A. (1985). Of Strategies, Deliberate and Emergent. Strategic Management Journal.

Paladino, B. y Williams, N. (2008). Moving Strategy Forward: Merging the Balanced Scorecard and Business Intelligence. Business Performance Management Magazine.

Porter, M. E. (1996). Las interrelaciones entre las unidades empresariales. Harvard Deusto business review.

Porter, M. E. (1996). What is a strategy? Harvard Business Review(November-December).

Porter, M. E. (2008). The Five Competitive Forces that Shape Strategy. Harvard business review.

Porter, M. y Siggelkow, N. (2008). Contextuality Within Activity Systems and Sustainability of Competitive Advantage. The Academy of Management Perspectives.

Raynor, M. E. (2007). What is Corporate Strategy, Really? Ivey Business Journal Online.

Scherpereel, C. M. (2006). Alignment: the duality of decision problems. Management Decision. 